\title{
Two Forms of Monomorphic Ventricular Tachycardia in a Patient with Brugada Syndrome
}

\author{
Shota Kakehashi, Tsukasa Kamakura, Takeshi Aiba and Kengo Kusano
}

\begin{abstract}
:
We herein report a 47-year-old man with relapsing polychondritis who developed monomorphic ventricular tachycardia (VT). His electrocardiogram in sinus rhythm showed a coved-type pattern, and there was no evidence of structural cardiac disease; therefore, he was diagnosed with Brugada syndrome. An electrophysiological study revealed a prolonged His-ventricular interval at the baseline. Two forms of VT were induced, which were shown to be bundle branch reentrant VT. A diagnosis of Brugada syndrome should not be ruled out in patients with monomorphic VTs, especially those with conduction abnormalities.
\end{abstract}

Key words: Brugada syndrome, ventricular tachycardia, bundle branch reentrant ventricular tachycardia

(Intern Med 59: 1287-1290, 2020)

(DOI: 10.2169/internalmedicine.4299-19)

\section{Introduction}

Brugada syndrome $(\mathrm{BrS})$ is an arrhythmogenic disease that causes sudden cardiac death mainly due to ventricular fibrillation (VF) or polymorphic ventricular tachycardia (VT). However, rare cases of monomorphic VT arising from a focal origin, such as the outflow tract, or due to re-entry involving the conduction system have also been reported $(1,2)$. Thus far, there have been no case reports of patients with $\mathrm{BrS}$ combined with multiple forms of monomorphic VT.

We herein report a patient with BrS who had two forms of monomorphic VT.

\section{Case Report}

A 47-year-old man with a history of relapsing polychondritis presented to our hospital with an episode of wide QRS tachycardia (195 beats/min) with a right bundle branch block (RBBB) pattern treated by cardioversion (Fig. 1A). No cardiac abnormality was observed on transthoracic echocardiography. Cardiac magnetic resonance imaging showed slight dilatation of the right ventricle (RV) (ratio of RV enddiastolic volume to body surface area: $106.8 \mathrm{~mL} / \mathrm{m}^{2}$ ); however, there was no RV wall motion abnormality, sign of fatty infiltration, or late gadolinium enhancement indicative of arrhythmogenic right ventricular cardiomyopathy (Fig. 1B). The histological findings on a myocardial biopsy of the right ventricle showed mild hypertrophy of myocytes and mild interstitial and perivascular fibrosis. However, there were no fibrofatty replacement. His baseline electrocardiogram (ECG) in high intercostal spaces showed a spontaneous type 1 pattern associated with QRS fragmentation; therefore, he was diagnosed with BrS (Fig. 1C).

His baseline PQ and QRS intervals were slightly prolonged (217 and $137 \mathrm{~ms}$, respectively). Genetic screening revealed that he carried an SCN5A variant (E1548K). His cousin died suddenly at 25 years old during sleep.

He underwent an electrophysiological study, revealing a prolonged His-ventricular interval of $80 \mathrm{~ms}$ at the baseline (Fig. 2A). Initially, VF was repeatedly induced by double extrastimuli from the RV apex. After the injection of a lowdose sodium channel blocker (12.5 mg pilsicainide), two different sustained VTs were induced (Fig. 2B, C): one was almost identical to the clinical wide QRS tachycardia with an RBBB pattern (Fig. 2B), and the other VT was a left bundle branch block (LBBB) pattern with left-axis deviation (Fig. 2C). The tachycardia with an RBBB pattern was diagnosed as bundle branch reentrant VT (BBRVT) based on the following findings: (1) atrio-ventricular dissociation, (2) His bundle electrograms $(\mathrm{H})$ preceded every ventricular electro- 
A

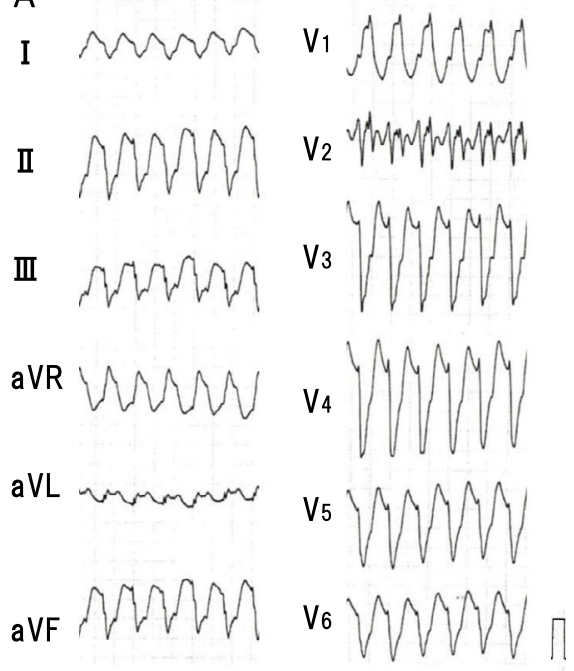

B

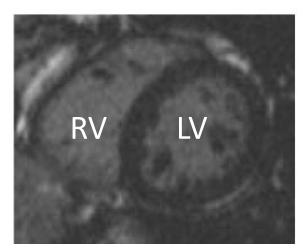

C

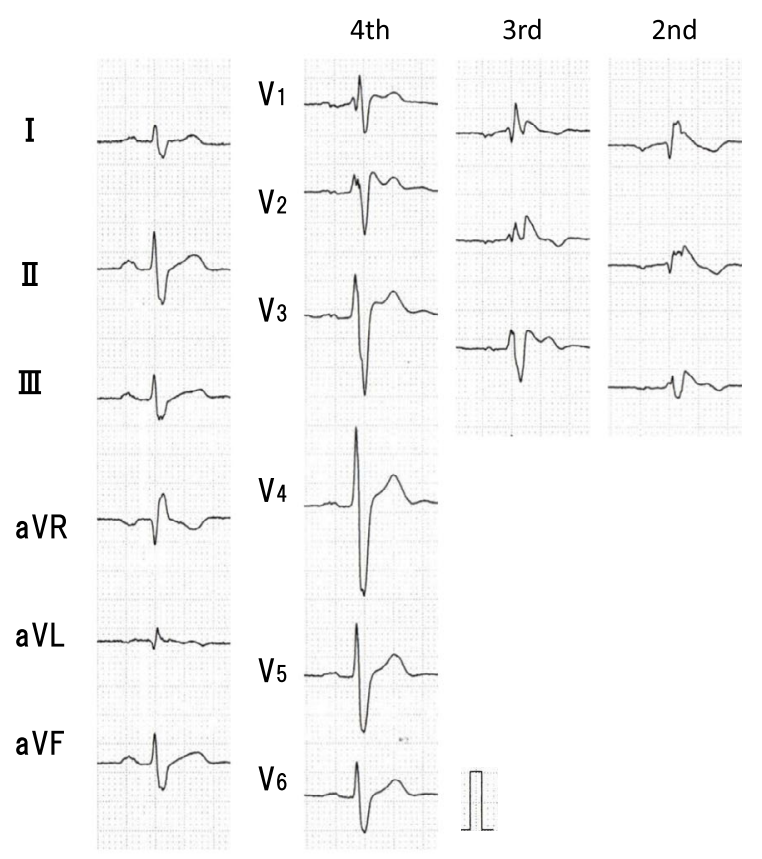

Figure 1. (A) Twelve-lead ECG during a spontaneous episode of VT. (B) Delayed enhancement cardiac magnetic resonance with a coronal view showed no late gadolinium enhancement. RV: right ventricle, LV: left ventricle. (C) The baseline ECG of this 47-year-old man. Type 1 ECG patterns were observed in leads $V_{1}$ and $V_{2}$ in the 2 nd intercostal space.

gram (V) with the $\mathrm{H}-\mathrm{V}$ interval greater than that recorded during sinus rhythm (Fig. 2D), and (3) spontaneous changes in $\mathrm{H}-\mathrm{H}$ intervals preceded similar changes in $\mathrm{V}-\mathrm{V}$ intervals (Fig. 2D). We were unable to perform a detailed electrophysiological study of VT with the LBBB pattern because only VT with the RBBB pattern was subsequently induced.

Proximal right bundle branch ablation during VT resulted in VT termination and rendered all VTs non-inducible. An ECG after ablation showed a coved-type pattern associated with the RBBB pattern (Fig. 3). He underwent transvenous implantable cardioverter-defibrillator implantation. He continued taking prednisolone for relapsing polychondritis. He remained free from monomorphic VT and VF without taking antiarrhythmic drugs during a follow-up period of 24 months. Transthoracic echocardiography 24 months after ablation showed no cardiac abnormality.

\section{Discussion}

To our knowledge, this is the first reported case of a $\mathrm{BrS}$ patient with two forms of VT. The diagnosis of very-earlystage arrhythmogenic right ventricular cardiomyopathy cannot be excluded; however, he showed no cardiac abnormalities indicative of the presence of arrhythmogenic right ventricular cardiomyopathy at diagnosis and during a 2-year follow-up period. In this case, clinical VT with an RBBB morphology was diagnosed as BBRVT, possibly with ante- grade conduction down the left bundle and retrograde conduction along the right bundle. Although a detailed electrophysiological study was not performed during another VT with LBBB pattern, this may have represented BBRVT with an opposite circuit, i.e. antegrade conduction down the right bundle and retrograde conduction along the left bundle, because ablation of the proximal right bundle branch rendered all VTs non-inducible.

BBRVT is characterized by macro re-entry within an abnormal His-Purkinje system. Although classically associated with structural heart disease such as dilated cardiomyopathy, BBRVT can occur in the absence of such disease as well. Recently, idiopathic BBRVT was reported in patients with mutations within genes implicated in conduction disease, such as SCN5A and LMNA (3). SCN5A mutations have been linked to BrS. It is known that BrS patients with $S C N 5 A$ mutation have a longer PQ interval and QRS duration than those without (4). We did not perform a functional analysis of the SCN5A variant identified in this case (E1548K); however, in silico prediction of the functional consequences of missense mutations using Polymorphism Phenotyping v2 predicted this variant to be possibly damaging, with a score of 0.902. The prolonged PQ and His-ventricular intervals as well as the Brugada-pattern ECG in this patient may have been due to the $S C N 5 A$ variant.

Relapsing polychondritis is another possible cause of the prolonged PQ and His-ventricular interval in this patient. Al- 

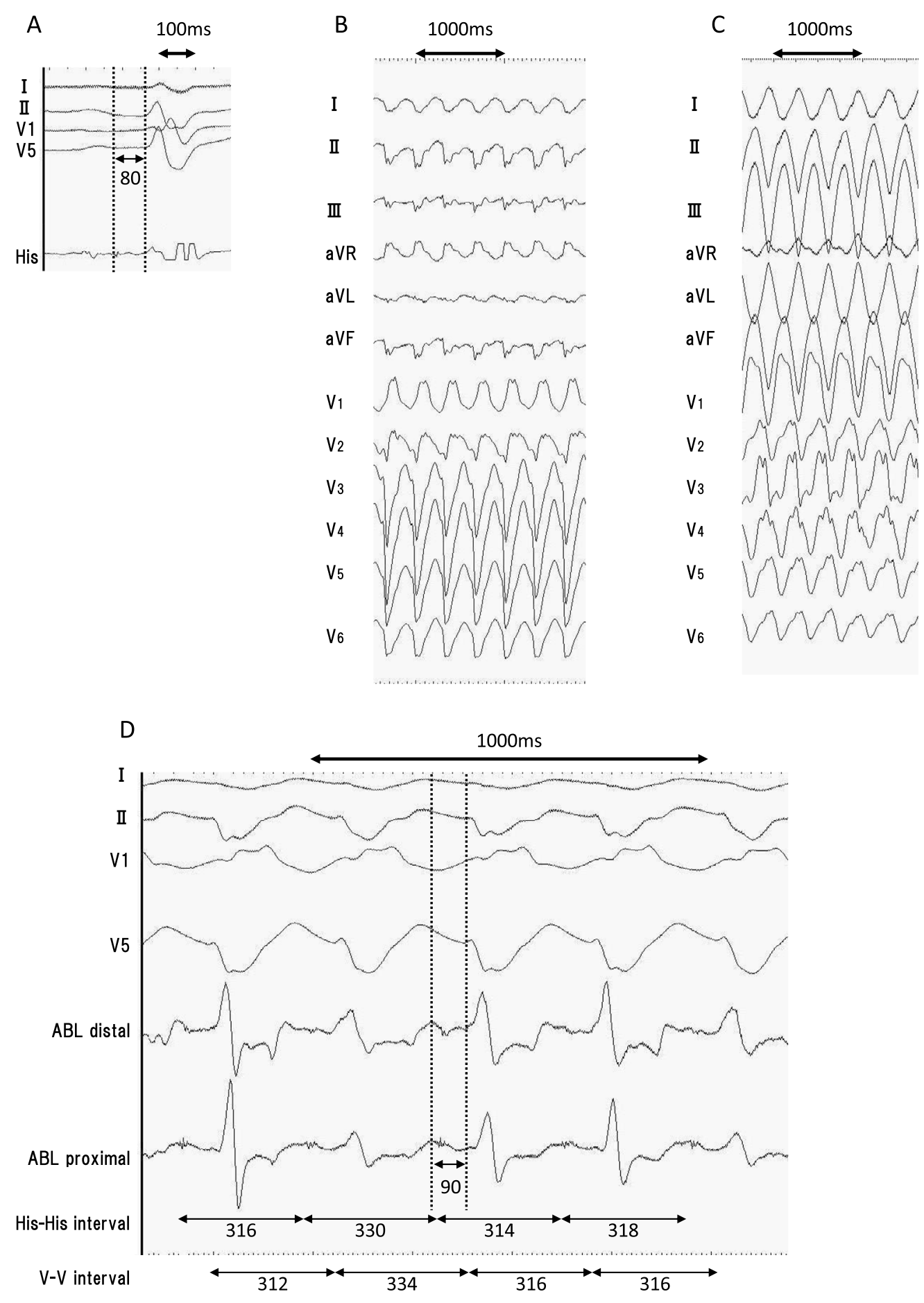

Figure 2. (A) Intracardiac recordings from the His bundle during an electrophysiological study. The His-ventricular interval was $80 \mathrm{~ms}$ during sinus rhythm. (B) A 12-lead ECG of induced tachycardia during an electrophysiological study with a right bundle branch block pattern. (C) A 12-lead ECG of induced tachycardia during an electrophysiological study with a left bundle branch block pattern. (D) Intracardiac recordings during ventricular tachycardia with the right bundle branch block pattern. An ablation catheter was placed at the His potential recording site at the base of the left ventricle. The His-ventricular interval was greater than that recorded during sinus rhythm $(90 \mathrm{~ms})$. Spontaneous changes in the His-His intervals preceded similar changes in the ventricular-ventricular intervals. V: ventricle

though relapsing polychondritis is a relatively rare multisystemic disease with recurrent inflammation, cardiac involvement is one of the major causes of mortality in this disease. A multicenter study of 62 polychondritis patients reported cardiac abnormalities in $22 \%$ of the patients (5). Of note, two patients had atrioventricular block, which suggests that relapsing polychondritis can cause cardiac conduction disturbance. Thus far, there have been no reports of relapsing 


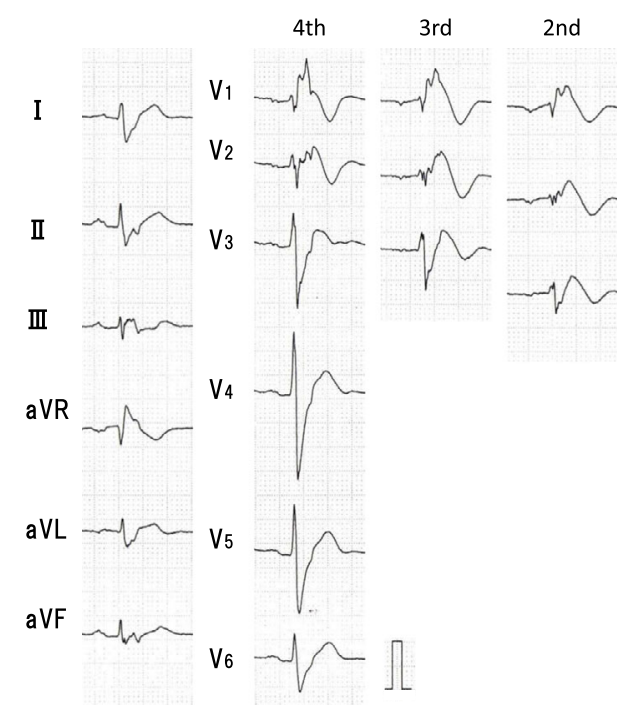

Figure 3. An ECG after ablation showed a coved-type pattern associated with right bundle branch block in lead $V_{2}$ in the standard position and in leads $\mathrm{V}_{1-3}$ in the second and third intercostal spaces.

polychondritis associated with $\mathrm{BrS}$. The association between relapsing polychondritis and $\mathrm{BrS}$ may be fortuitous in this case. Further studies are warranted to investigate the link between them.

In conclusion, we encountered a case of $\mathrm{BrS}$ associated with BBRVT. A diagnosis of BrS should not be ruled out in patients with monomorphic VTs, especially those with conduction abnormalities.
The authors state that they have no Conflict of Interest (COI).

\section{Financial Support}

This work was supported by the Japan Society for the Promotion of Science (JSPS) through a Grant-in-Aid for Scientific Research (KAKENHI Grant Number JP17K09545).

\section{References}

1. Rodriguez-Manero M, Sacher F, de Asmundis C, et al. Monomorphic ventricular tachycardia in patients with Brugada syndrome: a multicenter retrospective study. Heart Rhythm 13: 669-682, 2016.

2. Mazur A, Iakobishvili Z, Kusniec J, Strasberg B. Bundle branch reentrant ventricular tachycardia in a patient with the Brugada electrocardiographic pattern. Ann Noninvasive Electrocardiol 8: 352-355, 2003.

3. Roberts JD, Gollob MH, Young C, et al. Bundle branch re-entrant ventricular tachycardia: novel genetic mechanisms in a lifethreatening arrhythmia. JACC Clin Electrophysiol 3: 276-288, 2017.

4. Yamagata K, Horie M, Aiba T, et al. Genotype-phenotype correlation of SCN5A mutation for the clinical and electrocardiographic characteristics of probands with brugada syndrome: a Japanese multicenter registry. Circulation 135: 2255-2270, 2017.

5. Zeuner M, Straub RH, Rauh G, Albert ED, Scholmerich J, Lang B. Relapsing polychondritis: clinical and immunogenetic analysis of 62 patients. J Rheumatol 24: 96-101, 1997.

The Internal Medicine is an Open Access journal distributed under the Creative Commons Attribution-NonCommercial-NoDerivatives 4.0 International License. To view the details of this license, please visit (https://creativecommons.org/licenses/ by-nc-nd/4.0/).

(C) 2020 The Japanese Society of Internal Medicine Intern Med 59: 1287-1290, 2020 